\title{
Research on Antennas Alignment of Dynamic Point-to-Point Communication
}

\author{
Qilin Zeng $\left(\mathbb{D},{ }^{1,2}\right.$ Jiaxin Liu, ${ }^{1,2}$ and Weiming Xiong $\mathbb{C}^{1}$ \\ ${ }^{1}$ National Space Sciences Center, Chinese Academy of Sciences, Beijing 100190, China \\ ${ }^{2}$ University of Chinese Academy of Sciences, Beijing 100049, China \\ Correspondence should be addressed to Weiming Xiong; xwm@nssc.ac.cn
}

Received 12 April 2018; Revised 19 August 2018; Accepted 23 August 2018; Published 11 November 2018

Academic Editor: Ali Ramazani

Copyright (c) 2018 Qilin Zeng et al. This is an open access article distributed under the Creative Commons Attribution License, which permits unrestricted use, distribution, and reproduction in any medium, provided the original work is properly cited.

\begin{abstract}
In the dynamic point-to-point communication, to track and aim at antenna fast and accurately is the guarantee of high quality communication signal. In order to solve the problem of antenna alignment, we used the least square method (LSM) to fit the optimal level signal value (LSV) point which is based on coordinate coarse tracking alignment and matrix scanning strategy to find the LSV in this paper. Antenna is driven by two-dimensional turntable (azimuth and elevation angle (AE)): the twodimensional turntable is decomposed into two independent one-dimensional turntables, and the LSV in AE direction are obtained by scanning, respectively. The optimal LSV point of two-dimensional turntable can be find by combing optimal LSV point of two independent one-dimensional turntables. The method has the advantages of high precision and easy implementation and can meet the requirement of fast and accurately alignment in dynamic point-to-point communication antenna engineering.
\end{abstract}

\section{Introduction}

Directional antennas are commonly used for point-to-point communication in microwaves to reduce transmission and receiving power. The basic principle of alignment is that both stations can receive and transmit microwave signal. However, it is difficult to align the communication antenna at a long distance with the narrow beam angle of antenna, especially when there is relative motion at both stations. At present, the coordinate tracking control method (TCM) $[1,2]$ and the signal TCM $[3,4]$ (extreme tracking control) are mostly used to control the directional antenna alignment. Literature [5] proposed a joint two TCM, the antenna was controlled by the coordinate tracking method to reach the coarse alignment quickly, then the signal TCM was used to control the antenna to reach the beam fine alignment, and the antenna adjustment range is only within the main lobe of beam with using the signal TCM. In other words, it is only necessary to search for the maximum LSV within the coarse alignment errors range. However, due to noise and interference, these will result in the power value of the antenna alignment that may be less than the measurement power value of misalignment [6]. Therefore, the best LSV point is obtained by a single scan measurement that may be inaccurate. These will result in poor communication or even inability to establish communication links. Because of the limitation of measuring time, a good algorithm is needed to quickly realize the mutual alignment of two pairs of antenna beam centers. In this paper, we used the LSM [7] to fit the antenna optimal communication signal point of two stations within the coarse alignment errors range which is based on coordinate coarse tracking alignment and matrix scanning strategy to find the LSV.

In this paper, the problem of antenna tracking and alignment is analyzed under the movement of directional antenna for two stations; the solution and implementation scheme are proposed. The engineering method of antenna alignment is based on the known geographic latitude and longitude of both stations of communication, which is instructive for engineering realization of microwave communication antenna alignment.

\section{Processing Parameters}

2.1. Azimuth, Pitch Angle, and Distance Calculation. Both sides of the communication (two stations) obtain the local 


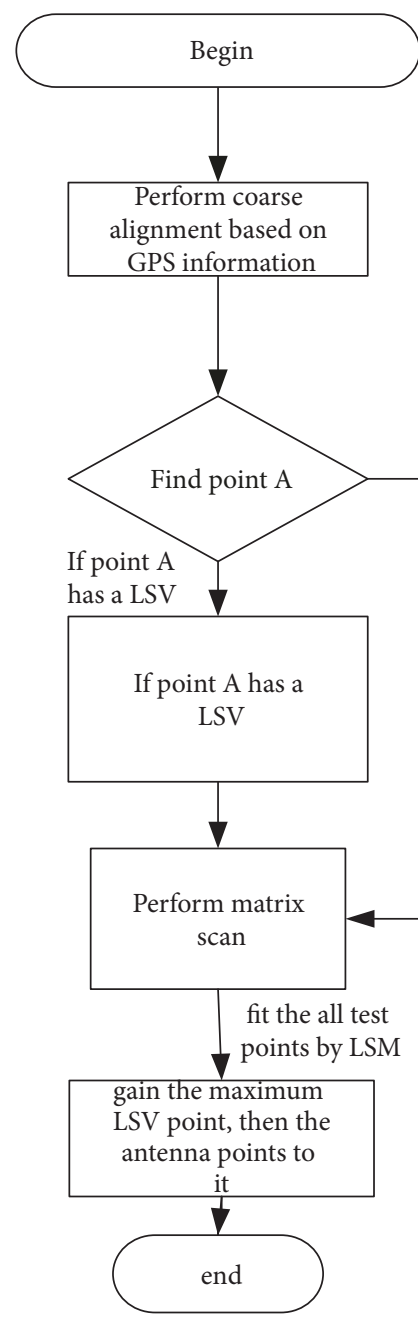

(a)work process

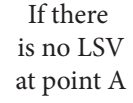

at point $\mathrm{A}$

Perform matrix scan until a LSV is found, this point as a point $\mathrm{A}$

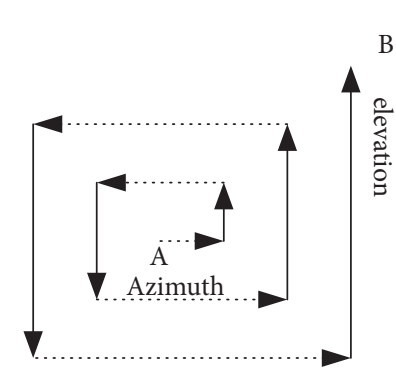

(b)Matrix scanning

FIGURE 1: Matrix scan for finding the optimal LSV.

longitude, latitude, and altitude data through GPS [8] devices. In order to facilitate the calculation and control of antenna tracking alignment angle, the AE of the antenna pointing to the other antenna should be calculated $[9,10]$ through GPS data. The AE of the antenna are measured by a local station angle sensor [11].

2.2. Matrix Scan and Find the Optimal LSV. The matrix scan is shown in Figure 1(b). The vertical line represents the search direction of elevation which is indicated by the solid black line. The horizontal represents the search direction of azimuth which is indicated by dotted line. The arrows represent the scan order. Point A shows the position of the coarse alignment in Figure 1(b). Figure 1(a) shows how to find the optimal LSV. If the LSV is not found at point A. We need to do a matrix scan until find the LSV and then set this point as point $A$. If the level signal value is found at point $A$, a matrix scan starts from point $A$ and finds all the value of level signal test points. According to the schematic diagram of Figure 1, the scan angle of $\mathrm{AE}$ is two times of the antenna $3 \mathrm{db}$ beam angle which taking point $\mathrm{A}$ as the origin, respectively. Point $B$ is the end point. If so, we can fit the all test points to find the maximum value of the level signal by LSM.

2.3. LSV Tracking Detection. Know the latitude and longitude of both stations communication platform, use the coordinate TCM for coarse alignment of the antenna, and then use the signal tracking method for high-precision secondary alignment, designers generally to monitor the strength of the receive signal by detecting the amplitude of the intermediate frequency signal. In this paper, the microwave signal theory to measure the strength of the receive LSV is written in Friis $[12,13]$ Equation in the following form:

$$
p(t)=p_{T} g_{T}(t) g_{R}(t)\left(\frac{\lambda}{4 \pi d_{s}}\right)^{2} \frac{1}{\rho}
$$

In (1), $p_{T}$ for transmit power, $g_{T}(t)$ is transmit antenna gain, $g_{R}(t)$ is receive antenna gain, $\rho$ is antenna power loss; $d_{s}$ is the communication distance between two stations; $\lambda$ is the electromagnetic wavelength. 
It can be known from (1) that the antenna gains will change, because of the different alignment angles of both stations. Substituting all parameters into (1), then calculate the receive power at both stations. From (1), it can be concluded that if the two antennas are aligned in a straight line, the SLV will be larger. Due to the existence of noise and interference, it is difficult to find out that the actual receive LSV is close to the theoretical LSV. Therefore, our paper proposes using the LSM to fit these receiving LSV points that get the optimal LSV point.

\section{Analysis and Simulation}

The LSM is a form of mathematical regression analysis that finds the line of optimal fit for a dataset, providing a visual demonstration of the relationship between the data points. We used the LSM fitting large amounts of data points by linear polynomial in the paper in which the method has proved that the distance errors between the fitted curve and the data point are the smallest [14]. In the process of point-topoint communication, to track and aim at antenna fast and accurately is the precondition of high quality communication signal. There will be big errors in the coarse alignment. Therefore, fine alignment is needed. It is only necessary to search the AE for the "power maximum" in the errors range of the coarse alignment. But the problem is that there are noise and interference, so the maximum power of any measurement has a certain errors. The feasible solution is to increase the number of measurements in the same angle of the antenna, and average these measurements. The measurement errors are linearly reduced with the increase of the square root of the measurement time. In this paper, the LSV in AE direction are obtained by scanning, respectively. The optimal LSV point of two-dimensional can be found by combing optimal LSV point of two independent one-dimensional turntables. Rotate the antenna to the optimal LSV point of AE, this point is the best communication location. The premise of the algorithm is that the measurement errors is Gaussian distribution [15]; it is clear that the actual communication noise is to meet the requirements. One of the advantages of LSM is that there is no requirement for the variance of measurement errors and measurement the uniformity of the coordinates. The algorithm can make full use of all measurement point information to fit the optimal LSV point.

Defining the alignment center of antenna as the optimal LSV point, the LSV can be expressed as a polynomial:

$$
p(\alpha, \beta, n)=-A r^{2}+p_{\max }+N_{n}
$$

$\alpha, \beta$ indicate the $\mathrm{AE}$ errors in the case of antenna alignment, respectively. $n$ represents the number of measurements. $A$ is the power drop coefficient, $r$ is the "distance" of $\mathrm{AE}$ errors, $r=\sqrt{\alpha^{2}+\beta^{2}}, p_{\max }$ indicates the receive power, and $N_{n}$ represents the measurement errors. Equation (2) shows that the measured LSV is maximum with the antenna alignment completely.

Equation (2) represents the optimal LSV of two-dimensional turntable. The two-dimensional turntables can be decomposed into two independent one-dimensional turntables. Equation (2) rewrites

$$
p(x, n)=-A_{x} x^{2}+p_{x \_ \text {max }}+N_{n}
$$

$x$ represents $\mathrm{AE}$ in (3); it represents the coefficient of the $\mathrm{x}$-coordinate.

If alignment center the antenna is in the position of $x=b$, $b$ is a constant and (3) can be written as

$$
p(x, n)=-A_{x}\left(x-x_{\text {center }}\right)^{2}+p_{x \_ \text {max }}+N_{n}
$$

According to the conventional polynomial, (4) can be expressed as

$$
y=a_{2} x^{2}+a_{1} x+a_{0}
$$

So we need to solve the value of $a_{2} / a_{1} / a_{0}$, which according to (5) can solve the center coordinates value of (4):

$$
x_{\text {center }}=-\frac{a_{1}}{2 a_{2}}
$$

According to the LSM principle

$$
A x=b
$$

Here $A$ is an $m \times n$ matrix.

According to (7)

$$
A^{T} A x=A^{T} b
$$

Equation (8) has a unique solution:

$$
\widehat{x}=\left(A^{T} A\right)^{-1} A^{T} b
$$

According to the multiple measured values of $x$ and $y$ and (5) the following equations can be derived:

$$
\begin{gathered}
a_{2} x_{1}{ }^{2}+a_{1} x_{1}+a_{0}=y_{1} \\
a_{2} x_{2}{ }^{2}+a_{1} x_{2}+a_{0}=y_{2} \\
\ldots \\
a_{2} x_{n}{ }^{2}+a_{1} x_{n}+a_{0}=y_{n}
\end{gathered}
$$

As a solution to the overdetermined equation of (10), calculate the values of $a, a_{1}, a_{2}$ by (9). The value of $a, a_{1}, a_{2}$ can be written as

$$
\begin{aligned}
{\left[\begin{array}{l}
a_{0} \\
a_{1} \\
a_{2}
\end{array}\right] } & =\left(\mathrm{V}^{\mathrm{T}} \mathrm{V}\right)^{-1} \mathrm{~V}^{\mathrm{T}} u \\
= & \left(\left[\begin{array}{ccc}
1 & \cdots & 1 \\
x_{1} & \cdots & x_{n} \\
x_{1}{ }^{2} & \cdots & x_{n}{ }^{2}
\end{array}\right]\left[\begin{array}{ccc}
1 & x_{1} & x_{1}{ }^{2} \\
\cdots & \cdots & \cdots \\
1 & x_{n} & x_{n}^{2}
\end{array}\right]\right)^{-1}
\end{aligned}
$$




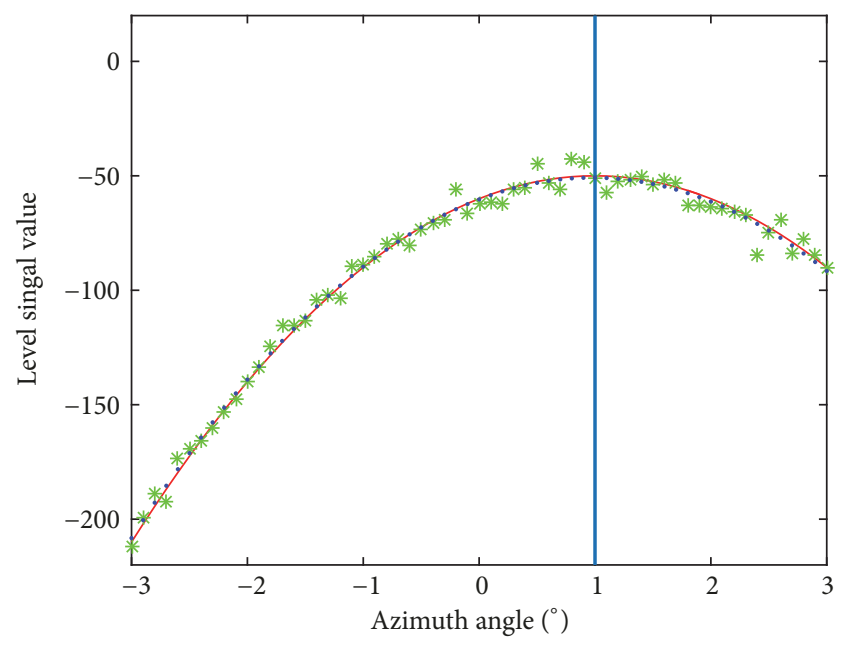

FIGURE 2: Fitting diagram of theoretical and noise measurement value.

$$
\begin{aligned}
& {\left[\begin{array}{ccc}
1 & \cdots & 1 \\
x_{1} & \cdots & x_{n} \\
x_{1}{ }^{2} & \cdots & x_{n}{ }^{2}
\end{array}\right]\left[\begin{array}{l}
y_{1} \\
\cdots \\
y_{n}
\end{array}\right] } \\
= & \left(\left[\begin{array}{ccc}
n & \sum x_{\mathrm{i}} & \sum x_{\mathrm{i}}^{2} \\
\sum x_{\mathrm{i}} & \sum x_{\mathrm{i}}^{2} & \sum x_{\mathrm{i}}^{3} \\
\sum x_{\mathrm{i}}^{2} & \sum x_{\mathrm{i}}^{3} & \sum x_{\mathrm{i}}^{4}
\end{array}\right]\right)^{-1}\left[\begin{array}{c}
\sum y_{\mathrm{i}} \\
\sum \mathrm{x}_{\mathrm{i}} y_{\mathrm{i}} \\
\sum \mathrm{x}_{\mathrm{i}}^{2} y_{\mathrm{i}}
\end{array}\right]
\end{aligned}
$$

In this paper, the AE direction is scanned within a certain errors range, respectively, fitting the optimal LSV of AE by LSM. Figure 2 shows the simulation result of theoretical measurement with noise and interference in azimuth angle. Elevation angle measurement is similar with azimuth angle, so this article will not repeat the simulation of elevation direction.

The theoretical center value of the antenna is set to $1 \mathrm{deg}$ in Figure 2. Simulation results of LSV by curve fitting of LSM are shown in Figure 2. As we can see from Figure 2, the green dots indicate the LSV of antenna scanning point, the blue dots represent the LSV points of correction by fitting, and the red curve is obtained by fitting of LSM. Through the simulation analysis, an interesting feature seen in Figure 2, it is found that the maximum value of the curve fitting is always around the abscissa of 1deg. The LSV reaches the maximum on the ordinate, approximately $-45.85 \mathrm{dbm}$. The fitted value is $1.0135 \mathrm{deg}$, which is very close to the theoretical center value 1deg. According to our simulation results, the optimal alignment point of antennas of both stations can be realized by curve fitting of LSM.

However, there may be some coincidence that one or several times optimal values are close to theoretical value by fitting of LSM. Therefore, our paper analyzed the value that the optimal points of 20 times and then compared these values with theoretical value. The light blue line is angle of the

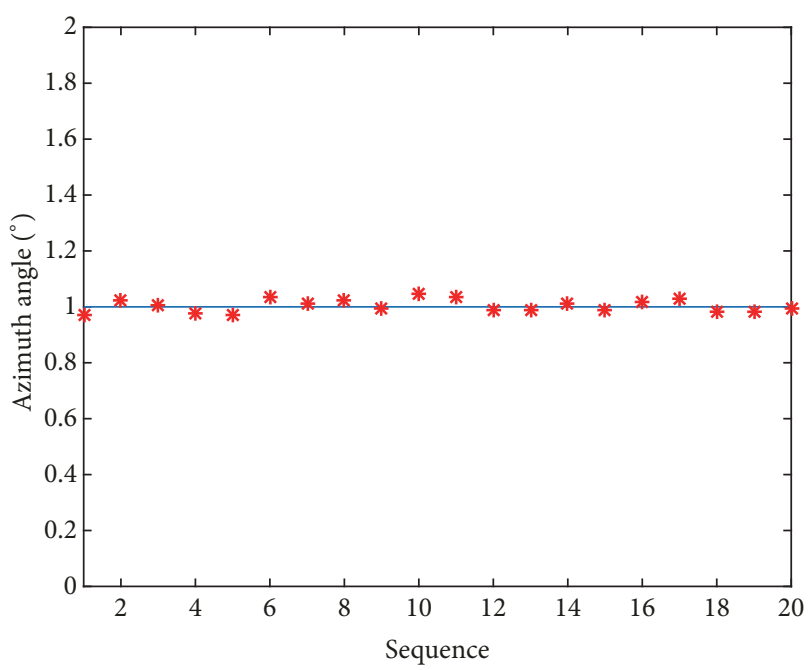

FIGURE 3: Analysis of actual measurements and theoretical measurements after multiple measurements.

theoretical center value of the antenna, and the 20 red dots are the different optimal value by multiplex fitting of LSM in Figure 3. As shown in Figure 3 it is found that the fitted value and the theoretical center value are always relatively close, and it can be seen from Figure 3 that the fitted dots always fluctuate slightly around the theoretical value center 1deg, although there are noise and interference. The simulation result shows that it is very reliable method to obtain the optimal value by fitting of LSM.

\section{Conclusion}

Because of the noise and interference in the dynamic pointto-point communication it is difficult to align the communication antenna at a long distance with the narrow beam angle of antenna. In this paper, a method based on coordinate tracking coarse alignment and matrix scanning to find the level is proposed; the LSM fitting is used to find the optimal LSV. Antenna is driven by two-dimensional turntable; the two-dimensional turntable is decomposed into two independent one-dimensional turntables, and the optimal LSV of AE direction is obtained, respectively. The simulation result shows that obtaining the optimal value by fitting of LSM is very reliable method. The method of LSM fitting can meet the requirement of fast alignment in antenna automatic tracking when two stations are stationary or in fast motion.

\section{Data Availability}

The data used to support the findings of this study are available from the corresponding author upon request.

\section{Conflicts of Interest}

The authors declare that they have no conflicts of interest. 


\section{References}

[1] Y. Zhang, X. Wang, and Y. Wang, "Research on automatic alignment technology of antennas in troposcatter communication vehicular stations," Radio Communications Technology, vol. 3, pp. 39-40, 2009.

[2] 1. Sheng, D. Yu, G. Li, and X. Ji, "Imitation human intelligence control-based antenna tracking system for vehicle," Computer Measurement \& Control, vol. 14, no. 2, pp. 212-213, 2006.

[3] D. Zhang, R. Sun, P. Li, and Z. Zhao, "Design of Servo tracking system of microwave vehicle antenna," Journal of Air Force Engineering University (Natural Science Edition), vol. 4, no. 3, pp. 47-49, 2003.

[4] X. Shen, Z. Qi, L. Wang, and G. Wang, "Research on direction of data conciliation antennas in location system using time difference among multi-station," Electronic Information Warfare Technology, vol. 23, no. 4, pp. 22-24, 2008.

[5] Z. Zhang and X. Li, "Research on antenna automatic control of dynamic point-to-point communication," Modern Defence Technology, vol. 4, article 018, 2013.

[6] M. I. Grace and D. A. Bradley, "Universal autoradar antenna alignment system,” Tech. Rep. US 6087995 A, 2000.

[7] C. L. Lawson and R. J. Hanson, Solving Least Squares Problems, vol. 15 of Classics in Applied Mathematics, Society for Industrial and Applied Mathematics (SIAM), Philadelphia, Pa, USA, 1995.

[8] A. Leick, L. Rapoport, and D. Tatarnikov, GPS Satellite Surveying, John Wiley \& Sons, 2015.

[9] J. Xiong, H. Zhu, and X. Huang, The Solution of Terrestrial Ellipsoid, Bayi Press, Beijing, China, 1993.

[10] Z. Li and G. Li, "Research on Rigorous algorithms for earth station azimuth and attitude," Radio Communications Technology, vol. 29, no. 6, pp. 30-32, 2003.

[11] Y. Zhu and L. Tan, "Design of intelligent system for searching an appointed oritentation based on digital compass HMR3000," Information Technology and Network Security, vol. 14, pp. 24-27, 2009.

[12] C. A. Balanis, Antenna Theory Analysis and Design, WileyInterscience, New York, NY, USA, 3rd edition, 2005.

[13] W. L. Stutzman and G. A. Thiele, Antenna Theory and Design, John Wiley and Sons, New York, NY, USA, 2nd edition, 1998.

[14] Y. Fei, Error Theory and Data Processing, Mechanical Industry Press, 1995.

[15] R. S. Chhikara and J. L. Folks, The Inverse Gaussian Distribution: Theory, Methodology, and Applications, Marcel Dekker, 1989. 


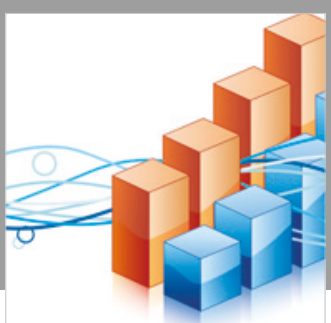

Advances in

Operations Research

\section{-n-m}
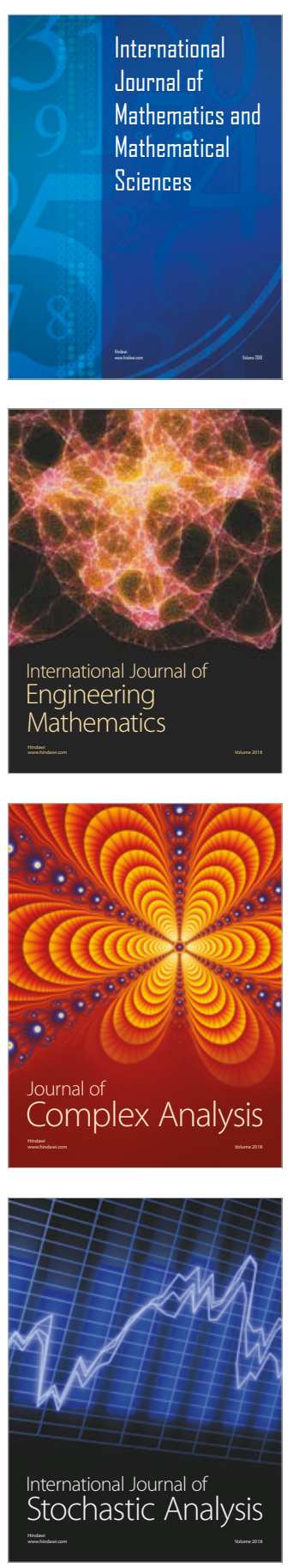
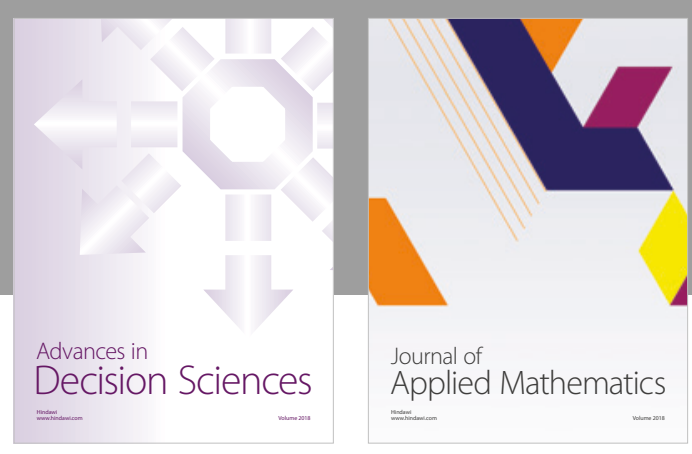

Journal of

Applied Mathematics
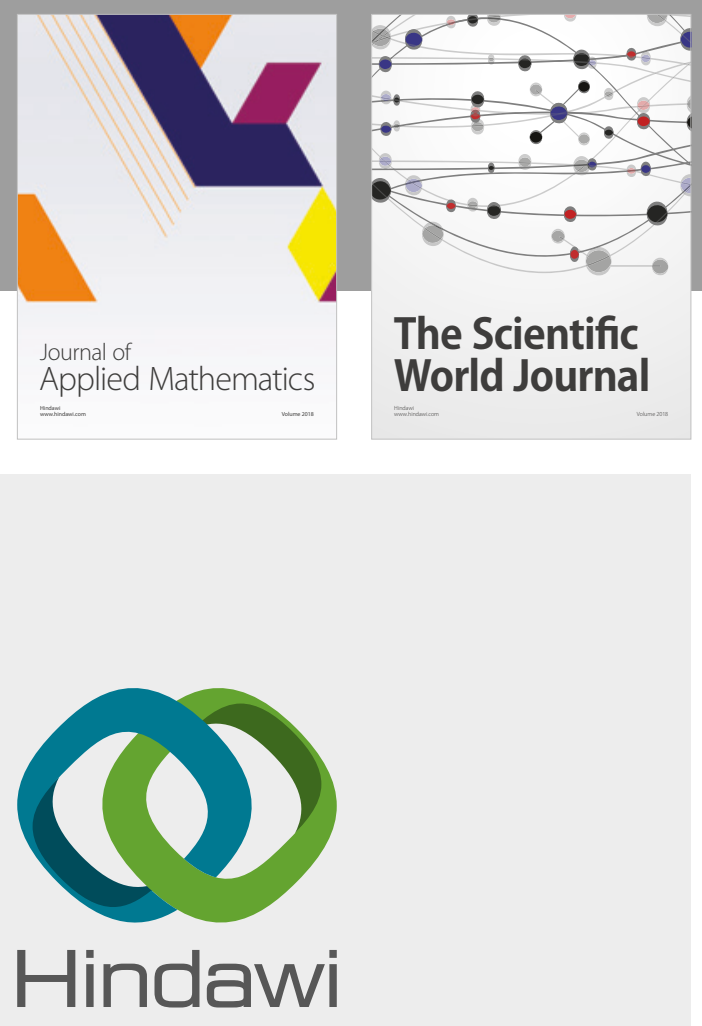

Submit your manuscripts at

www.hindawi.com

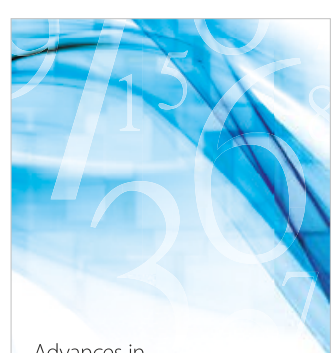

Advances in
Numerical Analysis
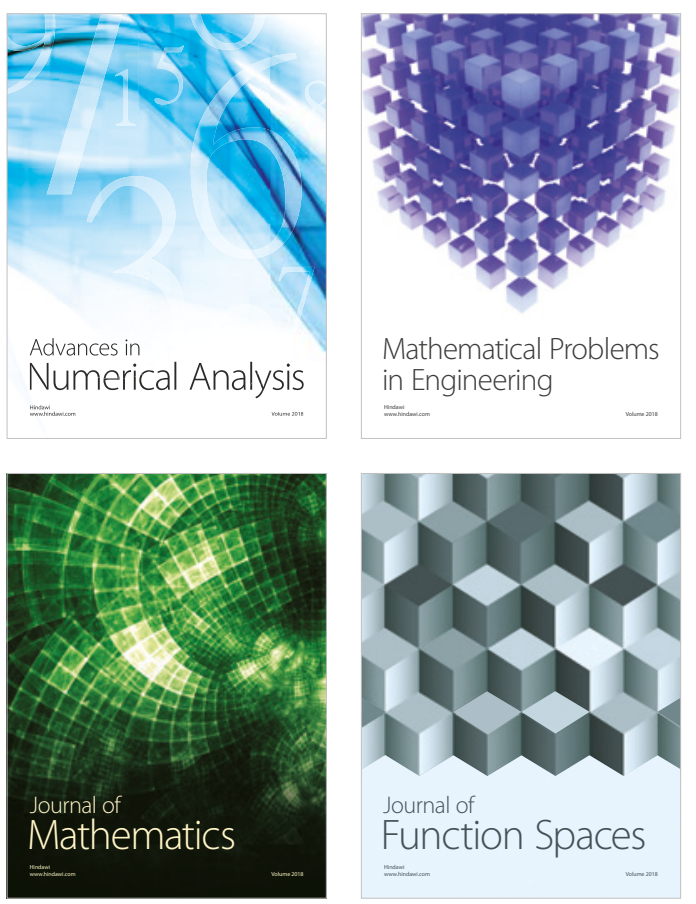

Mathematical Problems in Engineering

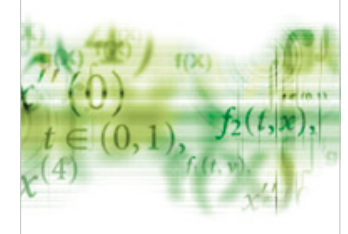

International Journal of

Differential Equations

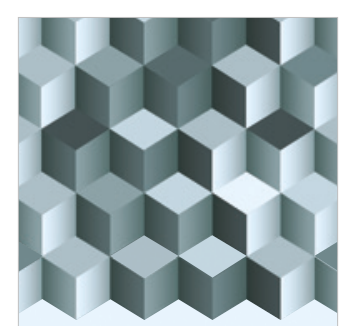

Journal of

Function Spaces

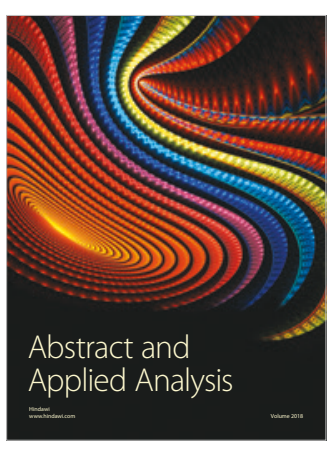

The Scientific

World Journal

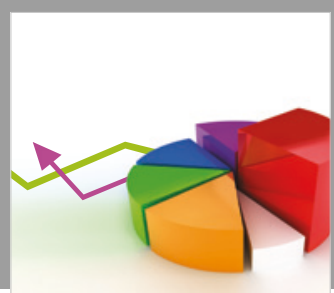

Journal of

Probability and Statistics
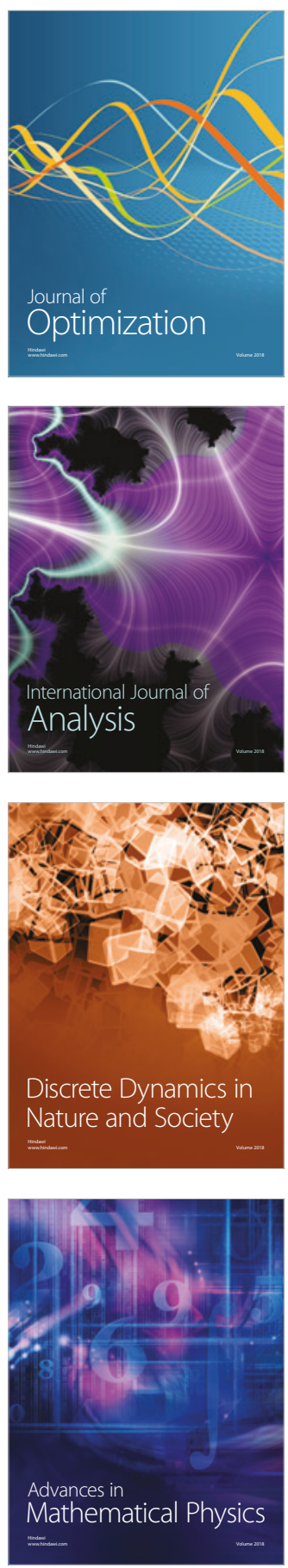